\title{
Multi-class ROC analysis from a multi-objective optimisation perspective
}

\author{
Richard M. Everson and Jonathan E. Fieldsend \\ Department of Computer Science, University of Exeter, Exeter, EX4 4QF, UK
}

\begin{abstract}
The Receiver Operating Characteristic (ROC) has become a standard tool for the analysis and comparison of classifiers when the costs of misclassification are unknown. There has been relatively little work, however, examining ROC for more than two classes. Here we discuss and present an extension to the standard twoclass ROC for multi-class problems.

We define the ROC surface for the $Q$-class problem in terms of a multi-objective optimisation problem in which the goal is to simultaneously minimise the $Q(Q-1)$ misclassification rates, when the misclassification costs and parameters governing the classifier's behaviour are unknown. We present an evolutionary algorithm to locate the Pareto front - the optimal trade-off surface between misclassifications of different types. The use of the Pareto optimal surface to compare classifiers is discussed and we present a straightforward multi-class analogue of the Gini coefficient. The performance of the evolutionary algorithm is illustrated on a synthetic three class problem, for both $k$-nearest neighbour and multi-layer perceptron classifiers.
\end{abstract}

Key words: receiver operating characteristic, evolutionary computation, multiple objectives, Pareto optimality, Gini coefficient.

\section{Introduction}

Classification or discrimination of unknown exemplars into two or more classes based on a 'training' dataset of examples, whose classification is known, is one

Email address: \{R.M.Everson, J.E.Fieldsend\}@exeter.ac.uk (Richard M. Everson and Jonathan E. Fieldsend).

1 This work was supported in part by the EPSRC, grant GR/R24357/01 
of the fundamental problems in supervised pattern recognition. Given a classifier that yields estimates of the exemplar's probability of belonging to each of the classes and when the relative relative costs of misclassification are known, it is straightforward to determine the decision rule that minimises the average cost of misclassification. If the cost of misclassification is taken to be 1 and there is no penalty for a correct classification then the optimal rule becomes: assign to the class with the highest posterior probability. In practical situations, however, the true costs of misclassification are unequal and frequently unknown or difficult to determine [e.g. Bradley, 1997; Adams and Hand, 1999]. In such cases the practitioner must either guess the misclassification costs or explore the trade-off in classification rates as the decision rule is varied.

Receiver Operating Characteristic (ROC) analysis provides a convenient graphical display of the trade-off between true and false positive classification rates for two class problems [Provost and Fawcett, 1997]. Since its introduction in the medical and signal processing literatures [Hanley and McNeil, 1982; Zweig and Campbell, 1993] ROC analysis has become a prominent method for selecting an operating point; see [Flach et al., 2003] and [Hernández-Orallo et al., 2004] for a recent snapshot of methodologies and applications.

In this paper we extend the spirit of ROC analysis to multi-class problems by considering the trade-offs between the misclassification rates from one class into each of the other classes. Rather than considering the true and false positive rates, we consider the multi-class ROC surface to be the solution of the multi-objective optimisation problem in which these misclassification rates are simultaneously optimised. Srinivasan [1999] has discussed a similar formulation of multi-class ROC, showing that if classifiers for $Q$ classes are considered to be points with coordinates given by their $Q(Q-1)$ misclassification rates, then optimal classifiers lie on the convex hull of these points. Here we describe the surface in terms of Pareto optimality and in section 3 we give an evolutionary algorithm for locating the optimal ROC surface when the classifier's parameters may be adjusted as part of the optimisation. Since multi-class ROC surfaces live in $Q(Q-1)$ dimensions visualisation is problematic, even for $Q=3$; in section 4 we therefore consider visualisation methods for ROC surfaces for a probabilistic $k$-nearest neighbour $(k$-nn) classifier [Holmes and Adams, 2002] and a multi-layer perceptron classifying synthetic data.

ROC analysis is frequently used for evaluating and comparing classifiers, the area under the ROC curve (AUC) or, equivalently, the Gini coefficient. Although the straightforward analogue of the AUC is unsuitable for more than two classes, in section 5 we develop a straightforward generalisation of the Gini coefficient which quantifies the superiority of a classifier's performance to random allocation. 


\section{ROC Analysis}

Here we describe the straightforward extension of ROC analysis to more than two classes (multi-class ROC) and draw some comparisons with the two class case.

In general a classifier seeks to allocate an exemplar or measurement $\mathbf{x}$ to one of a number of classes. Allocation of $\mathbf{x}$ to the incorrect class, say $\mathcal{A}_{j}$, usually incurs some, often unknown, cost denoted by $\lambda_{k j}$; we count cost a correct classification as zero: $\lambda_{k k}=0$. Denoting the probability of assigning an exemplar to $\mathcal{A}_{j}$ when its true class is in fact $\mathcal{A}_{k}$ as $p\left(\mathcal{A}_{j} \mid \mathcal{A}_{k}\right)$ the overall risk or expected cost is

$$
R=\sum_{j, k} \lambda_{k j} p\left(\mathcal{A}_{j} \mid \mathcal{A}_{k}\right) \pi_{k}
$$

where $\pi_{k}$ is the prior probability of $\mathcal{A}_{k}$. The performance of some particular classifier may be conveniently be summarised by a confusion matrix or contingency table, $\hat{C}$, which summarises the results of classifying a set of examples. Each entry $\hat{C}_{k j}$ of the confusion matrix gives the number of examples, whose true class was $\mathcal{A}_{k}$, that were actually assigned to $\mathcal{A}_{j}$. Normalising the confusion matrix so that each row sums to unity gives the confusion rate matrix, which we denote by $C$, whose entries are estimates of the misclassification probabilities: $p\left(\mathcal{A}_{j} \mid \mathcal{A}_{k}\right) \approx C_{k j}$. Thus the expected risk is estimated as

$$
R=\sum_{j, k} \lambda_{k j} C_{k j} \pi_{k}
$$

A slightly different perspective is gained by writing the expected risk in terms of the posterior probabilities of classification to each class. The conditional risk or average cost of assigning $\mathbf{x}$ to $\mathcal{A}_{j}$ is

$$
R\left(\mathcal{A}_{j} \mid \mathbf{x}\right)=\sum_{k} \lambda_{k j} p\left(\mathcal{A}_{k} \mid \mathbf{x}\right)
$$

where $p\left(\mathcal{A}_{k} \mid \mathbf{x}\right)$ is the posterior probability that $\mathbf{x}$ belongs to $\mathcal{A}_{k}$. If $\alpha(\mathbf{x})$ is a decision rule that assigns $\mathrm{x}$ to one of the classes $\mathcal{A}_{k}$, then expected overall risk associated with $\alpha$ is

$$
R=\int R(\alpha(\mathbf{x}) \mid \mathbf{x}) p(\mathbf{x}) d \mathbf{x} .
$$

The expected risk is then minimised, being equal to the Bayes risk, by assigning $\mathbf{x}$ to the class with the minimum conditional risk [e.g. Duda and Hart, 1973]. Choosing 'zero-one costs', $\lambda_{k j}=1-\delta_{k j}$, means that all misclassifications are equally costly and the conditional risk is equal to the class posterior prob- 
ability; one thus assigns to the class with the greatest posterior probability, which minimises the overall error rate.

When the costs are known it is therefore straightforward to make assignments to achieve the Bayes risk (provided, of course, that the classifier yields accurate assessments of the posterior probabilities $\left.p\left(\mathcal{A}_{k} \mid \mathbf{x}\right)\right)$. However, costs are frequently unknown and difficult to estimate, particularly when there are many classes; in this case it is useful to be able to compare the classification rates as the costs vary. For binary classification the conditional risk may be simply rewritten in terms of the posterior probability of assigning to $\mathcal{A}_{1}$, resulting in the rule: assign $\mathbf{x}$ to $\mathcal{A}_{1}$ if $P\left(\mathcal{A}_{1} \mid \mathbf{x}\right)>t=\lambda_{12} /\left(\lambda_{12}+\lambda_{22}\right)$. This classification rule reveals that there is, in fact, only one degree of freedom in the binary cost matrix and, as might be expected, the entire range of classification rates for each class can be swept out as the classification threshold $t$ varies from 0 to 1 . It is this variation of rates that the ROC curve exposes for binary classifiers. ROC analysis focuses on the classification of one particular class, say $\mathcal{A}_{1}$, and plots the true positive classification rate for $\mathcal{A}_{1}$ versus the false positive rate as the threshold $t$ or, equivalently, the ratio of misclassification costs is varied.

If more than one classifier is available (often produced by altering the parameters, w, of a particular classifier) then it can be shown that the convex hull of the ROC curves for the individual classifiers is the locus of optimum performance for that set of classifiers. [Provost and Fawcett, 1997, 1998] and Scott et al. [1998] have shown that performance at any point on the convex hull can be obtained by stochastically combining classifiers at the vertices of the convex hull.

Frequently in two class problems the focus is on a single class, for example, whether a set of medical symptoms are to be classified as benign or dangerous, so the ROC analysis practice of plotting of true and false positive rates for a single class has a direct physical interpretation. Also, since there are only three degrees of freedom in the binary confusion matrix, classification rates for the other class are easily inferred. Indeed, the confusion rate matrix, $C$ has only two degrees of freedom for binary problems. Focusing on one particular class is likely to be misleading when more than two classes are available for assignment. We therefore concentrate on the misclassification rates of each class to the others. In terms of the confusion rate matrix $C$ we consider the off-diagonal elements, the diagonal elements (i.e., the true positives) being determined by the off-diagonal elements since each row sums to unity.

With $Q$ classes there are $D=Q(Q-1)$ degrees of freedom in the confusion rate matrix and it is desirable to simultaneously minimise all the misclassification rates represented by these. For most problems, as in the binary problem, simultaneous optimisation will be impossible and some compromise between the various misclassification rates will have to be found. Knowledge of the costs 
makes this determination simple, but if the costs are unknown we propose to use multi-objective optimisation to discover the optimal trade-offs between the misclassifications rates.

Since the units in which costs are measured are immaterial, the costs may, without loss of generality, be taken as summing to unity. We assume here that there is zero cost for correct assignment, $\lambda_{i i}=0$, so there are $Q(Q-1)-1=$ $D-1$ degrees of freedom for the specification of costs. Consequently, the optimal trade-off surface between classification rates is, in general, of dimension $D-1$, one fewer than the dimension of the ambient space and separates the origin of the $[0,1]^{D}$ hypercube from the $(1, \ldots, 1)$ corner. Locally the surface is parameterised by the $(D-1)$ ratios of the misclassification costs in the same way that the binary ROC curve is parameterised by $\lambda_{12} /\left(\lambda_{12}+\lambda_{21}\right)$. To make these ideas more precise we now define the optimal ROC surface in terms of a Pareto front.

In general we will consider locating the optimal ROC surface as a function of the classifier parameters, w, as well as the costs; w might be, for example, the coefficients weighting the features in a linear discriminant or the weights of a neural network. For notational convenience and because they are treated as a single entity, we write the cost matrix $\boldsymbol{\lambda}$ and parameters as a single vector of generalised parameters, $\boldsymbol{\theta}=\{\boldsymbol{\lambda}, \mathbf{w}\}$; to distinguish $\boldsymbol{\theta}$ from the classifier parameters $\mathbf{w}$ we use the optimisation terminology decision vectors to refer to $\boldsymbol{\theta}$. We consider the $D$ misclassification rates to be functions (depending on the particular classifier) of the decision vectors, thus $C_{k j}=C_{k j}(\boldsymbol{\theta})$. The optimal trade-off between the misclassification rates is thus the defined by the minimisation problem:

$$
\text { minimise } C_{k j}(\boldsymbol{\theta}) \text { for all } k, j \text {. }
$$

If the all misclassification rates for one classifier with decision vector $\boldsymbol{\theta}$ are no worse than the classification rates for another classifier $\phi$ and at least one rate is better, then the classifier parameterised by $\boldsymbol{\theta}$ is said to strictly dominate that parameterised by $\boldsymbol{\phi}$. Thus $\boldsymbol{\theta}$ strictly dominates $\boldsymbol{\phi}$ (denoted $\boldsymbol{\theta} \prec \boldsymbol{\phi}$ ) iff:

$$
C_{j k}(\boldsymbol{\theta}) \leq C_{k j}(\boldsymbol{\phi}) \quad \forall k, j \quad \text { and } \quad C_{k j}(\boldsymbol{\theta})<C_{k j}(\boldsymbol{\phi}) \text { for some } k, j .
$$

Less stringently, $\boldsymbol{\theta}$ weakly dominates $\boldsymbol{\phi}$ (denoted $\boldsymbol{\theta} \preceq \boldsymbol{\phi}$ ) iff $C_{k j}(\boldsymbol{\theta}) \leq C_{k j}(\boldsymbol{\phi})$ $\forall k, j$.

A set $E$ of decision vectors is said to be non-dominated if no member of the set is dominated by any other member:

$$
\boldsymbol{\theta} \nprec \boldsymbol{\phi} \quad \forall \boldsymbol{\theta}, \boldsymbol{\phi} \in E .
$$


A solution to the minimisation problem (5) is thus Pareto optimal if it is not dominated by any other feasible solution, and the non-dominated set of all Pareto optimal solutions is known as the Pareto front. Recent years have seen the development of a number of evolutionary techniques based on dominance measures for locating the Pareto front; see Coello Coello [1999]; Deb [2001] and Veldhuizen and Lamont [2000] for recent reviews. Kupinski and Anastasio [1999] and Anastasio et al. [1998] introduced the use of multi-objective evolutionary algorithms (MOEAs) to optimise ROC curves for binary problems, illustrating the method on a synthetic data set and for medical imaging problems; and we have used a similar methodology for locating optimal ROC curves for safety-related systems [Fieldsend and Everson, 2004; Everson and Fieldsend, 2006]. In the following section we describe a straightforward evolutionary algorithm for locating the Pareto front for multi-class problems. We illustrate the method on a synthetic problem for two different classification models in section 4 .

\section{Locating multi-class ROC surfaces}

Here we describe a straightforward algorithm for locating the Pareto front for multi-class ROC problems using an analogue of mutation-based evolution. The procedure is based on the Pareto Archived Evolution Strategy (PAES) introduced by Knowles and Corne [2000]. In outline, the algorithm maintains a set or archive $E$ of decision vectors, whose members are mutually nondominating, which forms the current approximation to the Pareto front. As the computation progresses members of $E$ are selected, copied and their decision vectors perturbed, and the objectives corresponding to the perturbed decision vector evaluated; if the perturbed solution is not dominated by any element of $E$, it is inserted into $E$ and any members of $E$ which are dominated by the new entrant are removed. Therefore the archive can only move towards the Pareto front: it is in essence a greedy search where the archive $E$ is the current point of the search and perturbations to $E$ that are not dominated by the current $E$ are always accepted.

Algorithm 1 describes the procedure in more detail. The archive $E$ is initialised by evaluating the misclassification rates for a number (here 100) of randomly chosen parameter values and costs, and discarding those which are dominated by another element of the initial set. Then at each generation a single element, $\boldsymbol{\theta}$ is selected from $E$ (line 3 of Algorithm 1); selection may be uniformly random, but partitioned quasi-random selection (PQRS) [Fieldsend et al., 2003] was used here to promote exploration of the front. PQRS prevents clustering of solutions in a particular region of the front biasing the search because they are selected more frequently, thus increasing the efficiency and range of the search. 


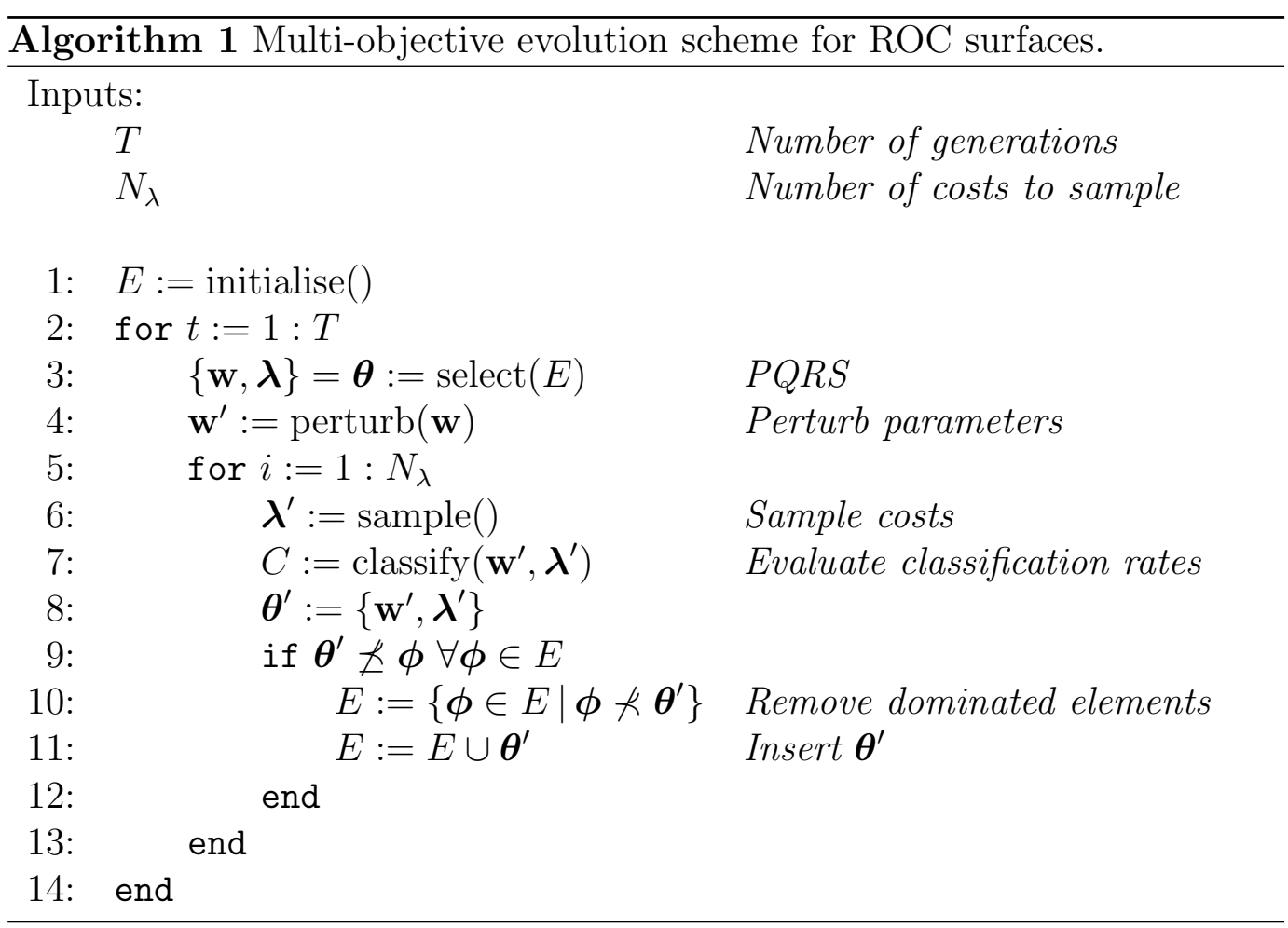

The selected parent decision vector is copied, after which the costs $\boldsymbol{\lambda}$ and classifier parameters $\mathbf{w}$ are treated separately. The parameters $\mathbf{w}$ of the classifier are perturbed or, in the nomenclature of evolutionary algorithms, mutated, to form a child, $\mathbf{w}^{\prime}$ (line 4). Here we seek to encourage wide exploration of parameter space by additively perturbing each of the parameters with a random number $\delta$ drawn from a heavy tailed distribution (such as the Laplacian density, $\left.p(\delta) \propto e^{-|\delta|}\right)$. The Laplacian distribution has tails that decay relatively slowly, thus ensuring that there is a high probability of exploring regions distant from the current solutions, facilitating escape from local minima [Yao et al., 1999].

With a proposed parameter set $\mathbf{w}^{\prime}$ on hand the procedure then investigates the misclassification rates as the costs are varied with fixed parameters. In order to do this we generate $N_{\lambda}$ sample costs, $\boldsymbol{\lambda}^{\prime}$, and evaluate the misclassification rates for each of them. Since the misclassification costs are non-negative and sum to unity, a straightforward way of producing samples is to make a draws from a Dirichlet distribution:

$$
\begin{aligned}
p(\boldsymbol{\lambda}) & =\operatorname{Dir}\left(\boldsymbol{\lambda} \mid \alpha_{1}, \ldots, \alpha_{D}\right) \\
& =\frac{\Gamma\left(\sum_{i=1}^{D} \alpha_{i}\right)}{\prod_{i=1}^{D} \Gamma\left(\alpha_{i}\right)}\left(1-\sum_{i=1}^{D-1} \lambda_{i}\right)^{\alpha_{D}-1} \prod_{i=1}^{D-1} \lambda_{i}^{\alpha_{i}-1}
\end{aligned}
$$

where the index $i$ labels the $D=Q(Q-1)$ off-diagonal entries in the cost matrix. As figure 1 illustrates, samples from a Dirichlet density lie on the simplex $\sum_{k j} \lambda_{k j}=1$. The $\alpha_{j k} \geq 0$ determine the density of the samples; since 


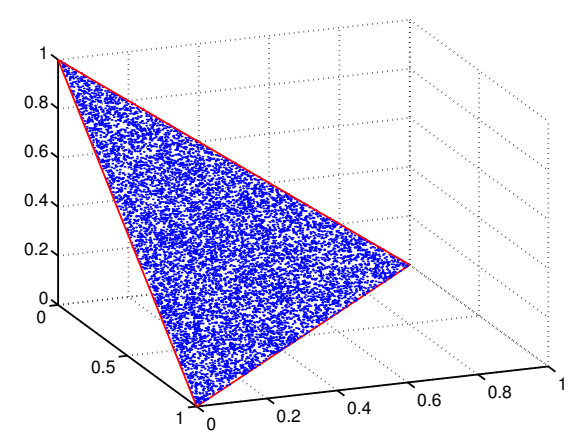

Figure 1. Samples from a 3-dimensional Dirichlet distribution, $\operatorname{Dir}(\boldsymbol{\lambda} \mid 1,1,1)$.

we have no preference for particular costs here, we set all the $\alpha_{k j}=1$ so that the simplex (that is, cost space) is sampled uniformly with respect to Lebesgue measure.

The misclassification rates for each cost sample $\boldsymbol{\lambda}^{\prime}$ and classifier parameters $\mathbf{w}$ are used to make class assignments for each example in the given dataset (line 7). Usually this step consists of merely modifying the posterior probabilities $p\left(\mathcal{A}_{k} \mid \mathbf{x}\right)$ to find the assignment with the minimum expected cost and is therefore computationally inexpensive as the probabilities need only be computed once for each $\mathbf{w}^{\prime}$. The misclassification rates $C_{k j}\left(\boldsymbol{\theta}^{\prime}\right)(j \neq k)$ comprise the objective values for the decision vector $\boldsymbol{\theta}^{\prime}=\left\{\mathbf{w}^{\prime}, \boldsymbol{\lambda}\right\}$ and decision vectors that are not dominated by any member of the archive $E$ are inserted into $E$ (line 11) and any decision vectors in $E$ that are dominated by the new entrant are removed (line 10). We remark that this algorithm, unlike the original PAES algorithm, uses an archive whose size is unconstrained, permitting better convergence [Fieldsend et al., 2003]. Although at first sight unrestricted archives may appear to be computationally costly to store and query (as implied by lines 9 and 10), for realistic problems on modern machines storage is not constraining and logarithmic time queries and updates can be achieved by the use of special data structures [Fieldsend et al., 2003]; in practice the main computational burden is usually evaluating the classification rates.

A $(\mu+\lambda)$ evolution strategy (ES) is defined as one in which $\mu$ decision vectors are selected as parents at each generation and perturbed to generate $\lambda$ offspring. ${ }^{2}$ The set of offspring and parents are then truncated or replicated to provide the $\mu$ parents for the following generation. Although Algorithm 1 is based on a $(1+1)$-ES, it is interesting to note that each parent $\boldsymbol{\theta}$ is perturbed to yield $N_{\lambda}$ offspring, all of whom have the classifier parameters $\mathbf{w}^{\prime}$ in common. With linear costs, evaluation of the objectives for many $\boldsymbol{\lambda}^{\prime}$ samples is inexpensive. Nonlinear costs could be incorporated in a straightforward manner, although it would necessitate complete reclassification for each $\boldsymbol{\lambda}^{\prime}$ sample and it would therefore be more efficient to resample $\mathbf{w}$ with each $\boldsymbol{\lambda}^{\prime}$.

\footnotetext{
$\overline{2}$ We adhere to the optimisation terminology for $(\mu+\lambda)$-ES, although there is a potential for confusion with the costs $\lambda_{k j}$.
} 


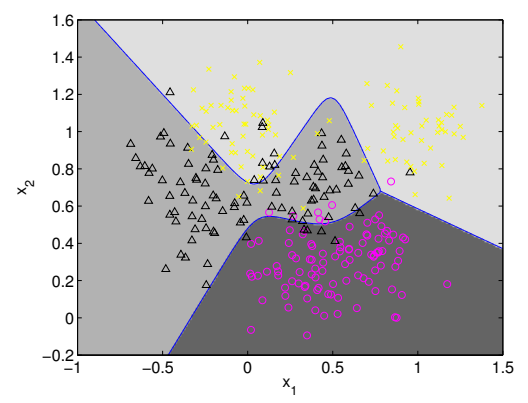

Figure 2. Synthetic 3-class data. Magenta circles mark class 1, black triangles class 2 and yellow crosses class 3. Blue lines mark the optimal decision boundaries for equal misclassification costs.

\section{Illustrations}

In this section we illustrate the performance of the evolutionary algorithm on synthetic data, which is readily understood. Subsequently we give results for a number of standard multi-class problems. For simplicity we use two relatively simple classifiers, the $k$-nearest neighbour classifier $(k$-nn), which we now briefly describe in its probabilistic form [Holmes and Adams, 2002], and the multi-layer perceptron (MLP), a standard neural network.

\subsection{Synthetic data}

In order to gain an understanding of the Pareto optimal ROC surface for multiple class classifications we extend a two-dimensional, two-class synthetic data set devised by Ripley [1994] by adding additional Gaussian functions corresponding to an additional class. The resulting data set comprises 3 classes, the conditional density for each being a mixture of two Gaussians. Covariance matrices for all the components were isotropic: $\boldsymbol{\Sigma}_{j}=0.3 \mathbf{I}$. Denoting by $\boldsymbol{\mu}_{j i}$ for $i=1,2$ the means of the two Gaussian components generating samples for class $j$, the centres were located at:

$$
\begin{aligned}
\boldsymbol{\mu}_{11}=(0.7,0.3)^{T} & \boldsymbol{\mu}_{12}=(0.3,0.3)^{T} \\
\boldsymbol{\mu}_{21}=(-0.7,0.7)^{T} & \boldsymbol{\mu}_{22}=(0.4,0.7)^{T} \\
\boldsymbol{\mu}_{31}=(1.0,1.0)^{T} & \boldsymbol{\mu}_{32}=(0.0,1.0)^{T}
\end{aligned}
$$

Each component had equal mixing weight 1/6. The 300 samples used here, together with the equal cost Bayes optimal decision boundaries, are shown in Figure 2 . 


\subsection{Probabilistic k-nn}

One of the most popular methods of statistical classification is the $k$-nearest neighbour model $(k-\mathrm{nn})$. The method has a ready statistical interpretation, and has been shown to have an asymptotic error rate no worse than twice the Bayes error rate [Cover and Hart, 1967]. The method is essentially geometrical, assigning the class of an unknown exemplar to the class of the majority of its $k$ nearest neighbours in some training data. More precisely, in order to assign a datum $\mathbf{x}$, given known classes and examples in the form of training data $\mathcal{D}=\left\{y_{n}, \mathbf{x}_{n}\right\}_{n=1}^{N}$, the $k$-nn method first calculates the distances $d_{i}=\left\|\mathbf{x}-\mathbf{x}_{i}\right\|$. If the $Q$ classes are a priori equally likely, the probability that $\mathbf{x}$ belongs to the $j$-th class is then evaluated as $p\left(\mathcal{A}_{j} \mid \mathbf{x}, k, \mathcal{D}\right)=k_{j} / k$, where $k_{j}$ is the number of the $k$ data points with the smallest $d_{n}$ belonging to $\mathcal{A}_{n}$.

Holmes and Adams [2002, 2003] have extended the traditional $k$-nn classifier by adding a parameter $\beta$ which controls the 'strength of association' between neighbours. The posterior probability of $\mathbf{x}$ belonging to each class $\mathcal{A}_{j}$ is given by the predictive likelihood:

$$
p\left(\mathcal{A}_{j} \mid \mathbf{x}, k, \beta, \mathcal{D}\right)=\frac{\exp \left[\beta \sum_{\mathbf{x}_{n} \sim \mathbf{x}}^{k} u\left(d\left(\mathbf{x}, \mathbf{x}_{n}\right)\right) \delta_{j y_{n}}\right]}{\sum_{q=1}^{Q} \exp \left[\beta \sum_{\mathbf{x}_{n} \sim \mathbf{x}}^{k} u\left(d\left(\mathbf{x}, \mathbf{x}_{n}\right)\right) \delta_{q y_{n}}\right]} .
$$

Here $\delta_{m n}$ is the Kronecker delta and $\sum_{\mathbf{x}_{n} \sim \mathbf{x}}^{k}$ means the sum over the $k$ nearest neighbours of $\mathbf{x}$ (excluding $\mathbf{x}$ itself). If the non-increasing function of distance $u(\cdot)=1 / k$, then the term $\sum_{\mathbf{x}_{n} \sim \mathbf{x}}^{k} u\left(d\left(\mathbf{x}_{n}, \mathbf{x}\right)\right) \delta_{j y_{n}}$ counts the fraction of the $k$ nearest neighbours of $\mathbf{x}$ in the same class $j$ as $\mathbf{x}$. In the work reported here we choose $u$ to be the tricube kernel which gives decreasing weight to distant neighbours [Fan and Gijbels, 1996].

Holmes \& Adams use the probabilistic formulation of the $k$-nn classifier as part of a Bayesian scheme in which they average over the parameters $k$ and $\beta$. Here we regard $\mathbf{w}=\{k, \beta\}$ as parameters to be adjusted as part of Algorithm 1 as the Pareto optimal ROC surface is sought.

To discover the Pareto optimal ROC surface, the optimisation algorithm was run for $T=10000$ proposed parameter values, with $N_{\lambda}=100$, resulting in an estimated Pareto front comprising approximately 7500 mutually nondominating parameter and cost combinations; we judge that the algorithm is very well converged and obtain very similar results by permitting the algorithm to run for only $T=2000$ generations.

There are $D=Q(Q-1)=6$ objectives to be minimised and, in common with other high-dimensional optimisation problems, visualisation of the 5dimensional Pareto front is important for understanding the trade-offs possible. A discussion of advanced visualisation techniques for this can be found in 

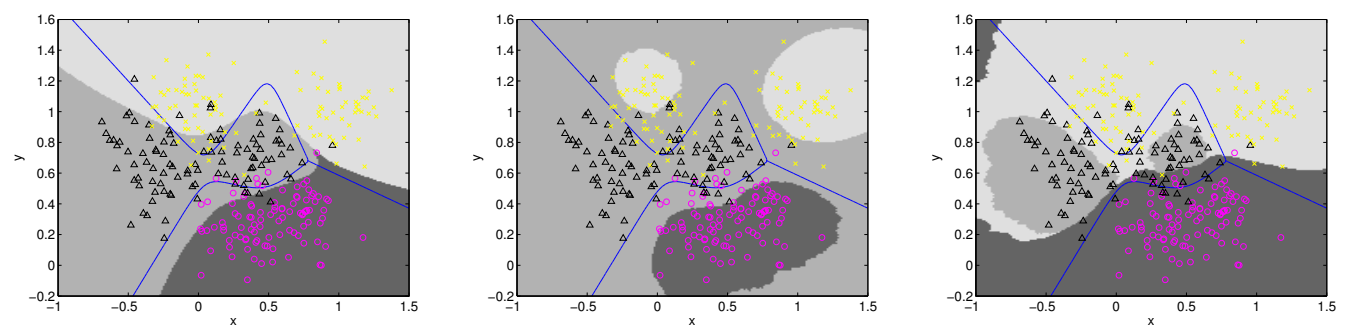

Figure 3. Decision regions for various $k$-nn classifiers on multi-class ROC surface. Grey scale background shows the class to which a point would be assigned. Blue lines show the ideal equal-cost decision boundary. Symbols show actual training data. Left: Parameters corresponding to minimum total misclassification error on the training data. Middle: Decision regions corresponding to the minimum $C_{21}$ and $C_{23}$ and conditioned on this, minimum $C_{31}$ and $C_{13}$. Right: Decision regions corresponding to minimising $C_{12}$ and $C_{32}$.

Fieldsend and Everson [2005b]

The left panel of the figure shows the decision regions that yield the smallest total misclassification error, 32/300. This point has very similar decision regions to the equal-cost Bayes optimal ones, as might be expected since the overlap between classes is approximately comparable and there are equal numbers in each class. There are (3 in the results presented here) other parameterisations and cost combinations that also achieve this minimum misclassification rate.

By contrast with the decision regions which are optimal for roughly equal costs, the middle and right panels of Figure 3 show decision regions for imbalanced costs. The middle panel shows a decision region corresponding to minimising $C_{21}$ and $C_{23}$ : this, of course, can be achieved by setting $\lambda_{21}$ and $\lambda_{23}$ to be large, so that every $\mathcal{A}_{2}$ example (black triangle) is correctly classified, no matter what the cost. For these data there are many decision regions correctly classifying every $\mathcal{A}_{2}$ and we display the decision regions that also minimise $C_{31}$ and $C_{13}$. For these data, it is possible to make $C_{31}=C_{13}=0$ because $\mathcal{A}_{1}$ and $\mathcal{A}_{3}$ are adjacent only along a boundary distant from $\mathcal{A}_{2}$ points; such complete minimisation will in general not be possible. Of course, the penalty to be paid for minimising the $\mathcal{A}_{2}$ rates together with $C_{31}$ and $C_{13}$ is that $C_{32}$ and $C_{12}$ are large: in fact, $C_{32}>C_{12}$.

The right panel of Figure 3 shows the reverse situation: here the costs for misclassifying either $\mathcal{A}_{1}$ or $\mathcal{A}_{3}$ as $C_{2}$ are high. With these data, although not in general, of course, it is possible to reduce $C_{12}$ and $C_{32}$ to zero, as shown by the decision regions which ensure that $\mathcal{A}_{2}$ examples (black triangles) are only classified correctly when it does not result in incorrect assignment of the other two classes to $\mathcal{A}_{2}$. In this case the greatest misclassification rate is $C_{23}$ (black triangles as yellow crosses). 


\subsection{Multi-layer perceptron classifiers}

We also used a multi-layer perceptron (MLP) with a single hidden layer with 5 units and softmax output units as the classifier optimised by Algorithm 1. Again, the algorithm was run for $T=10000$ evaluations of the classifier, resulting in an estimated Pareto front or ROC surface comprising approximately 4800 mutually non-dominating parameter and cost combinations. Note that for the MLP the parameter vector $\mathbf{w}$ consists of 33 weights and biases in contrast to just two parameters for the $k$-nn classifier. In this case the archive was initialised by training a single MLP using quasi-Newton optimisation of the data likelihood [e.g. Bishop, 1995] which finds a point on or near the Pareto front corresponding to equal misclassification costs; subsequent iterations of the evolutionary algorithm are therefore largely concerned with exploring the Pareto front rather than locating it. Figure 4 shows decision regions for points on the Pareto front corresponding to those shown for the $k$-nn classifier in Figure 3.

Decision regions corresponding to minimum overall misclassification error (Figure 4, left) are similar to those for the $k$-nn classifier. The additional flexibility inherent in the MLP with 33 adjustable parameters permits the decision regions to be more finely tuned to the data: for example, the $\mathcal{A}_{2}$ (black triangles) $\mathcal{A}_{3}$ (yellow crosses) boundary in Figure 4 lies to the right of the $\mathcal{A}_{2}$ data point at $(-0.456,1.21)$ so that it is correctly classified in contrast to the $k$-nn decision regions in Figure 3. Although no explicit measures were taken to prevent over-fitting, the decision boundaries on the front are quite smooth and do not exhibit signs of over-fitting; permitting the optimisation algorithm to run for very long times might lead to over-fitting but we have not encountered it in the work reported here.

MLP decision regions minimising $C_{21}, C_{23}, C_{31}$ and $C_{13}$, shown in the middle panel of Figure 4 are similar to the $k$-nn regions (Figure 3 ) where the data density is high, but differ in detail where data are sparse. The same is true of the decision regions minimising misclassifications as $\mathcal{A}_{2}$, as can be seen by comparing the right panels of Figures 3 and 4.

The decision regions illustrated in the middle and right panels of Figures 3 and 4 may thought of as lying on the edges of the Pareto surface because they correspond to one or more objectives being exactly minimised. These points are the analogues of the extreme ends of the usual two-class ROC curve where the false positive rate or the true positive rate is extremised. The curvature of the ROC curve in these regions is generally small, signifying that large changes in the costs yield large changes in either the true or false positive rate, but only small changes in the other. We observe a similar behaviour here: quite large changes the $\lambda_{k j}$ in these regions yield quite small changes in the all the 

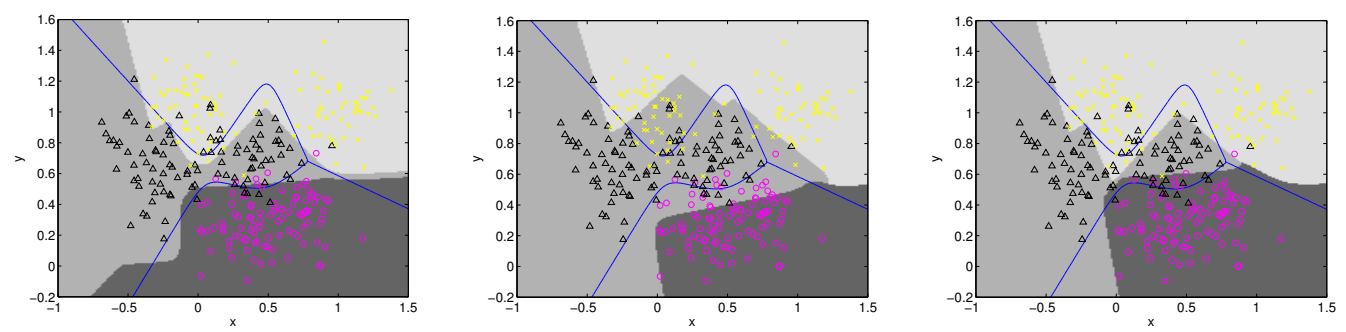

Figure 4. Decision regions for various MLP classifiers on multi-class ROC surface. Grey scale background shows the class to which a point would be assigned. Blue lines show the ideal equal-cost decision boundary. Symbols show actual training data. Left: Parameters corresponding to minimum total misclassification error on the training data. Middle: Decision regions corresponding to the minimum $C_{21}$ and $C_{23}$ and conditioned on this, minimum $C_{31}$ and $C_{13}$. Right: Decision regions corresponding to minimising $C_{12}$ and $C_{32}$.

misclassification rates except the one which has been extremised, suggesting that the curvature of the Pareto surface is low in these areas.

A common use of the two-class ROC curve is to locate a 'knee', a point of high curvature. The parameters at the knee are chosen as the operational parameters because the knee signifies the transition from rapid variation of true positive rate to rapid variation of false positive rate. Methods for numerically calculating the curvature of a manifold from point sets in more than two dimensions are, however, not well developed (for a discussion, see Fieldsend and Everson [2005a]). Initial investigations in this direction have so far yielded only very crude approximations to the curvature in the 6-dimensional objective space and we refrain from displaying them here. Although direct visual inspection of the curvature for multi-class problems is presently infeasible, we draw attention to the fact that the evolutionary algorithm yields a Pareto front of classifier parameterisations and associated costs.

\subsection{False positive rates}

Humans are particularly adept at visualisation in two and three dimensions, the intrinsic dimensions of the world we inhabit, and relatively inept at visualisation of high-dimensional objects. It is tempting therefore to attempt to reduce the dimension of the ROC surface sought so as to permit visualisation. One straightforward way to achieve is to locate the trade-off surface for minimising misclassifications into each class, that is the false positive rate for each class. We minimise the $Q$ objectives

$$
F_{k}(\mathbf{w}, \boldsymbol{\lambda})=\sum_{j \neq k} C_{k j} \quad k=1, \ldots, Q
$$




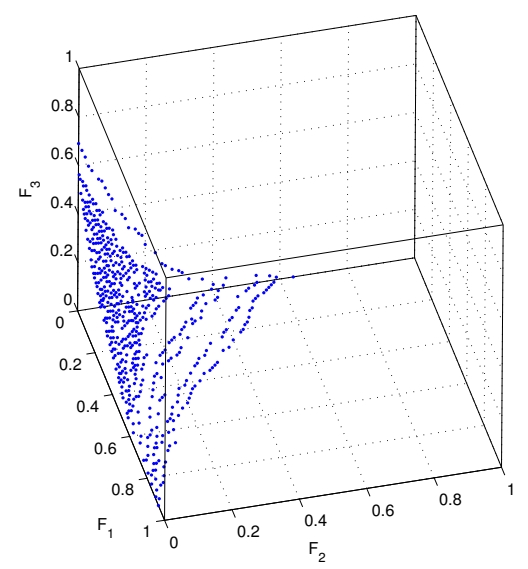

Figure 5. Estimated Pareto front where the objectives are the overall misclassification rates for each class. Synthetic data using $k$-nn classifiers.

The evolutionary algorithm is easily adapted to this minimisation problem and Figure 5 shows the Pareto surface obtained for the synthetic data, using the $k$-nn classifier and running the optimiser for $T=10^{4}$ generations. We call this front the 'false positive rate front' (similar to the true positive front described by Mossman [1999]). The figure shows the tradeoff between false positive rates for each of the three classes and a 'corner' or 'knee' can be located where all three misclassification rates are small and approximately equal. Decision regions for parameterisations close to the corner are similar to the equal-costs Bayes decision regions (Figure 2) and correspond to cost matrices, $\boldsymbol{\lambda}$, with approximately equal entries. As may be expected the false positive rate for one class may be reduced, but, as the surface shows, only at the expense of raising the false positive rate for the other classes.

The false positive rate Pareto front is easily visualised (at least for three class problems), but information on exactly how misclassifications are made is lost. However, the full $D$-dimensional Pareto surface may usefully be viewed in 'false positive space'. Figure 6 shows the solutions on the estimated Pareto front obtained using the full $Q(Q-1)$ objectives for the $k$-nn classifier, but each solution is plotted as a grey scale symbol at the coordinate given by the $Q=3$ false positive rates (12), with the symbol grey scale denoting the class into which the greatest number of misclassifications are made. ${ }^{3}$ Although the solutions obtained by optimising the false positive rates directly lie on the full Pareto surface (in $Q(Q-1)$ dimensions) the converse is not true and the projections into false positive space do not form a surface. Nonetheless, at least for these data, they lie close to a surface, which aids visualisation and navigation of the full Pareto front. The relation between the solutions on the full Pareto front and the false positive rate front is made more precise as

$\overline{3}$ A colour movie showing views from other angles can be found at http://www. dcs.ex.ac.uk/ reverson/research/mcroc. 

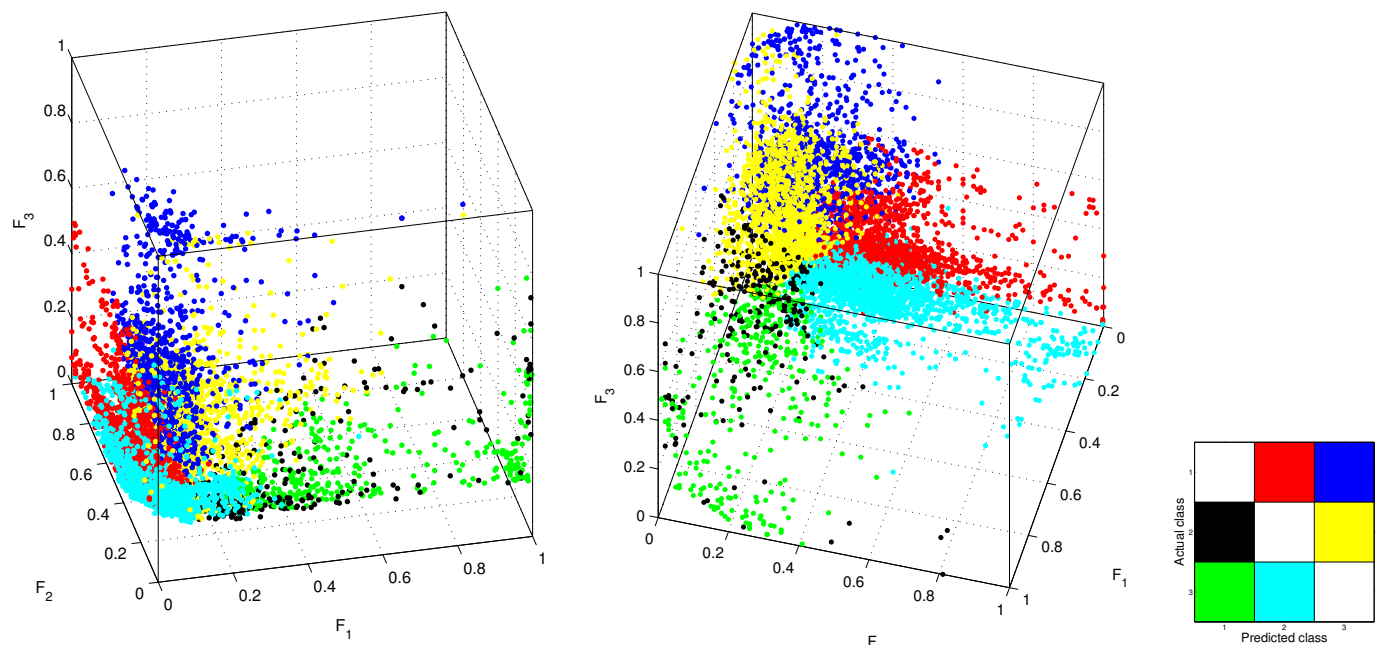

Figure 6. Two views of the estimated Pareto front for synthetic data classified with a $k$-nn classifier viewed in false positive space. Axes show the false positive rates for each class and points are coloured according to the class into which the greatest number of misclassifications are made, the overall misclassification rates for each class.

follows. If $E$ is a set of $Q(Q-1)$-dimensional solutions lying in the full Pareto front, let $E_{Q}$ be the set of $Q$-dimensional vectors representing the false positive coordinates of elements of $E$. The extremal set of non-dominated elements of $E_{Q}$ is

$$
\tilde{E}_{Q}=\left\{\mathbf{f} \in E_{Q} \mid \mathbf{f} \nprec \mathbf{f}^{\prime} \in E_{Q}\right\} .
$$

Then solutions in $\tilde{E}_{Q}$ also lie in the false positive rate front.

\section{Comparing classifiers}

In two class problems the area under the ROC curve (AUC) is often used to compare classifiers. As clearly explained by Hand and Till [2001], the AUC measures a classifier's ability to separate two classes over the range of possible costs and is linearly related to the Gini coefficient. In this section we compare the $k$-nn and MLP classifiers using a measure based on the volume dominated by the Pareto optimal ROC surface.

By analogy with the AUC, we might therefore use the volume of the $Q(Q-1)$ dimensional hypercube that is dominated by elements of the ROC surface for classifier $X$ as a measure of $X$ 's performance. In binary and multi-class problems alike its maximum value is 1 when $X$ classifies perfectly. If the classifier allocates at random, the ROC surface is the simplex in $Q(Q-1)$-dimensional space with vertices at length $Q-1$ along each coordinate vector. The volume 


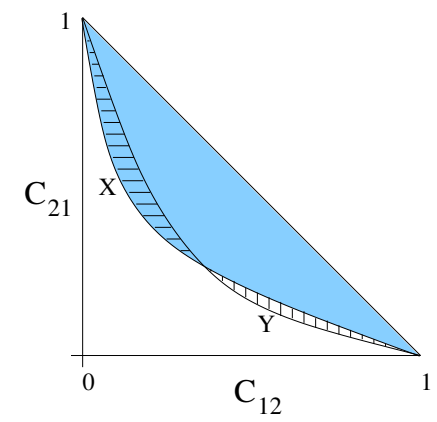

Figure 7. Illustration of the $G$ and $\delta$ measures for $Q=2$. The shaded area denotes $G(X)$, the horizontally dashed area denotes $\delta(X, Y)$ and the vertically dashed area denotes $\delta(Y, X)$.

of the unit hypercube dominated by this can be derived as follows: First we note that the volume of the pyramidal region between the origin and the simplex with vertices at a distance $L$ along each coordinate vector is $\frac{L^{Q(Q-1)}}{(Q(Q-1)) !}$. The volume lying between the origin and the random allocation simplex is, therefore:

$$
\frac{(Q-1)^{Q(Q-1)}}{(Q(Q-1)) !}
$$

Only part of this volume lies in the unit hypercube however, as the corners (excluding that at the origin) relate to infeasible regions where classification rates are $>1$. Each of these $Q(Q-1)$ corner regions is also a pyramidal volume, but with sides of length $Q-2$. The total volume of the region between the origin and the random allocation simplex which also lies in the unit hypercube is therefore

$$
\frac{(Q-1)^{Q(Q-1)}}{(Q(Q-1)) !}-\frac{Q(Q-1)(Q-2)^{Q(Q-1)}}{(Q(Q-1)) !} .
$$

We denote this region by $P$. When $Q=2$ the second term in equation 15 is zero so that the total volume (area) between the origin and the random allocation simplex is just $1 / 2$. This corresponds to the area under the diagonal in a conventional ROC plot (although binary ROC plots are usually made in terms of true positive rates versus false positive rates for one class, the false positive rate for the other class is just 1 minus the true positive rate for the other class). However, when $Q>2$, the volume not dominated by the random allocation simplex is very small; even when $Q=3$, the volume not dominated is $\approx 0.0806$. We therefore define $G(X)$ to be the analogue of the Gini coefficient in two dimensions, namely the proportion of the volume of the $Q(Q-1)$-dimensional unit hypercube that is dominated by elements of the ROC surface, but is not dominated by the simplex defined by random allocation (as illustrated by the blue shaded area in Figure 7 for the $Q=2$ case). In binary classification problems this corresponds to twice the area between the ROC curve and the 

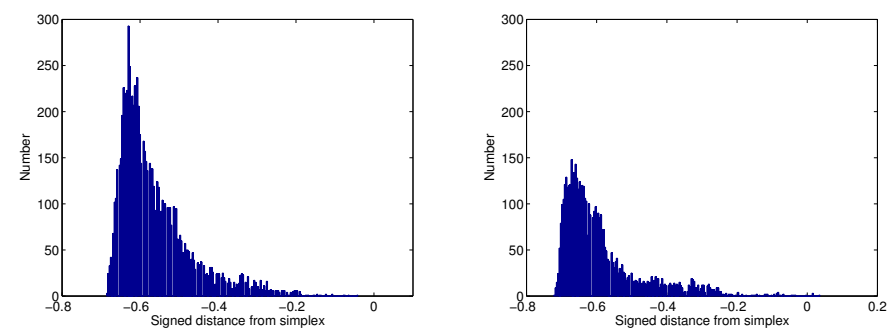

\begin{tabular}{|c|c|}
\hline$G(k-\mathrm{nn})$ & 0.916 \\
\hline$G(\mathrm{MLP})$ & 0.970 \\
\hline$\delta(k-\mathrm{nn}, \mathrm{MLP})$ & 0.0001 \\
\hline$\delta(\mathrm{MLP}, k-\mathrm{nn})$ & 0.054 \\
\hline
\end{tabular}

Figure 8. Distances from the random classifier simplex. Negative distances correspond to models in P. Left: $k$-nn; Middle: MLP. Right: Generalised Gini coefficients and exclusively dominated volume comparisons of the $k$-nn and MLP classifiers.

diagonal. In multi-class problems $G(X)$ quantifies how much better $X$ is than random allocation. It can be simply estimated by Monte Carlo sampling of $P$ in the unit hypercube.

If every point on the optimal ROC surface for classifier $X$ is dominated by a point on the ROC surface for classifier $Y$, then classifier $Y$ has a superior performance to classifier $X$. In general, however, neither ROC surface will completely dominate the other: regions of $X$ 's surface, $R_{X}$, will be dominated by regions of $Y$ 's and vice versa; in binary problems this corresponds to ROC curves that cross. To quantify the classifier's relative performance we therefore define $\delta(X, Y)$ to be the volume of $P$ that is dominated by elements of $R_{X}$ and not by elements of $R_{Y}$ (marked in Figure 7 with horizontal lines). Note that $\delta(X, Y)$ is not a metric because although it is non-negative it is not symmetric. Also if $R_{X}$ and $R_{Y}$ are subsets of the same non-dominated set $W$, (i.e., $R_{X} \subseteq W$ and $\left.R_{Y} \subseteq W\right)$, then $\delta(X, Y)$ and $\delta(Y, X)$ may have a range of values depending on their precise composition; see Fieldsend et al. [2003] for more details. Situations like this are rare in practice, however, and measures like $\delta$ have proved useful for comparing Pareto fronts.

The right panel of Figure 8 shows $G$ and $\delta$ calculated from $10^{5}$ Monte Carlo samples for the $k$-nn and MLP classifiers. The MLP ROC surface dominates a larger proportion of the volume and, as the $\delta$ measures show, almost every point on the $k$-nn ROC surface is weakly dominated by a point on the MLP surface. As mentioned above, the MLP has 33 adjustable parameters compared with 2 for $k$-nn, so it is unsurprising that the MLP front almost completely dominates the $k$-nn front.

It should be noted that not all of the classifiers located by the evolutionary algorithm lie in the volume $P$. This occurs because in multi-class problems, performance on one misclassification rate may be sacrificed to be worse than random in order to obtain superior performance on the other rates. In fact all of the $k$-nn models and all but 4 of $\approx 4800$ MLP models on the ROC surface lie in $P$. Figure 8 shows histograms of the signed distances of classifiers on the ROC surface from the random allocation simplex; negative distances correspond to 
classifiers in $P$. Clearly the majority of classifiers lie some distance closer to the origin than the random allocation simplex. In the absence of additional information or preferences (such as a maximum misclassification rate that can be tolerated for a particular class), a method of selecting the optimal classifier is to choose the one most distant from the random allocation simplex, a practice that corresponds to the selecting the classifier most distant from the diagonal on binary ROC plots.

\section{Conclusion}

In this paper we have considered multi-class generalisations of ROC analysis from a multi-objective optimisation perspective. Consideration of the role of costs in classification leads to a multi-objective optimisation problem in which misclassification rates are simultaneously optimised. The resulting trade-off surface generalises the binary classification ROC curve because on it one misclassification rate cannot be improved without degrading at least one other. We have presented a straightforward general evolutionary algorithm which efficiently locates approximations to the Pareto optimal ROC surfaces.

An appealing quality of the ROC curve is that it can be plotted in two dimensions and an operating point selected from the plot. Unfortunately, the dimension of the Pareto optimal ROC surface grows as the square of the number of classes, which hampers visualisation. Projection into 'false positive space' is an effective visualisation method for 3-class problems as the false positive rates summarise the gross overall performance, allowing further analysis of exactly which classes are misclassified into which to be focused in particular regions. Nonetheless, it is likely that problems with more than three classes will require some a priori assessment of important trade-offs because of the difficulty in interpreting 16 or more competing rates. Reliable calculation and visualisation of the curvature of the ROC surface will be important for selecting operating points; current work is focused on this area.

The Pareto optimal ROC surface yields a natural way of comparing classifiers in terms of the volume that the classifiers' ROC surfaces dominate. We defined and illustrated a generalisation of the Gini coefficient for multi-class problems that quantifies the superiority of a classifier to random allocation. Finally, we remark that some imprecise information about the costs of misclassification may often be available; for example the approximate bounds on the ratios of the $\lambda_{k j}$ may be known. In this case the evolutionary algorithm is easily focused on the relevant region by setting the Dirichlet parameters $\alpha_{k j}$ appearing in (8) to be in the ratio of the expected costs, with their magnitudes setting the variance in the cost ratios. 


\section{References}

N.M. Adams and D.J. Hand. Comparing classifiers when the misallocation costs are uncertain. Pattern Recognition, 32:1139-1147, 1999.

M. Anastasio, M. Kupinski, and R. Nishikawa. Optimization and FROC analysis of rule-based detection schemes using a multiobjective approach. IEEE Transactions on Medical Imaging, 17:1089-1093, 1998.

C. Bishop. Neural Networks for Pattern Recognition. Clarendon Press, Oxford, 1995.

A.P. Bradley. The use of the area under the ROC curve in the evaluation of machine learning algorithms. Pattern Recognition, 30:1145-1159, 1997.

C.A. Coello Coello. A Comprehensive Survey of Evolutionary-Based Multiobjective Optimization Techniques. Knowledge and Information Systems. An International Journal, 1(3):269-308, 1999.

T.M. Cover and P.E. Hart. Nearest neighbor pattern classification. IEEE Transactions on Information Theory, 13(1):21-27, 1967.

K. Deb. Multi-Objective Optimization Using Evolutionary Algorithms. Wiley, Chichester, 2001.

R.O. Duda and P.E. Hart. Pattern Classification and Scene Analysis. Wiley, New York, 1973.

R.M. Everson and J.E. Fieldsend. Multi-objective optimisation of safety related systems: An application to short term conflict alert. IEEE Transactions on Evolutionary Computation, 2006. (In press).

J.Q. Fan and I. Gijbels. Local polynomial modelling and its applications. Chapman \& Hall, London, 1996.

J.E. Fieldsend and R.M. Everson. ROC Optimisation of Safety Related Systems. In J. Hernández-Orallo, C. Ferri, N. Lachiche, and P. Flach, editors, Proceedings of ROCAI 2004, part of the 16th European Conference on Artificial Intelligence (ECAI), pages 37-44, 2004.

J.E. Fieldsend and R.M. Everson. Formulation and comparison of multi-class ROC surfaces. In Proceedings of the 2nd ROC Analysis in Machine Learning Workshop, part of the 22nd International Conference on Machine Learning (ICML 2005), pages 41-48, 2005a.

J.E. Fieldsend and R.M. Everson. Visualisation of multi-class ROC surfaces. In Proceedings of the 2nd ROC Analysis in Machine Learning Workshop, part of the 22nd International Conference on Machine Learning (ICML 2005), pages 49-56, 2005b.

J.E. Fieldsend, R.M. Everson, and S. Singh. Using Unconstrained Elite Archives for Multi-Objective Optimisation. IEEE Transactions on Evolutionary Computation, 7(3):305-323, 2003.

P. Flach, H. Blockeel, C. Ferri, J. Hernández-Orallo, and J. Struyf. Decision support for data mining: Introduction to ROC analysis and its applications. In D. Mladenic, N. Lavrac, M. Bohanec, and S. Moyle, editors, Data Mining and Decision Support: Integration and Collaboration, pages 81-90. Kluwer, 2003. 
D.J. Hand and R.J. Till. A simple generalisation of the area under the ROC curve for multiple class classification problems. Machine Learning, 45:171$186,2001$.

J.A. Hanley and B.J. McNeil. The meaning and use of the area under a receiver operating characteristic (ROC) curve. Radiology, 82(143):29-36, 1982.

J. Hernández-Orallo, C. Ferri, N. Lachiche, and P. Flach, editors. ROC Analysis in Artificial Intelligence, 1st International Workshop, ROCAI-2004, Valencia, Spain, 2004.

C.C. Holmes and N.M. Adams. A probabilistic nearest neighbour method for statistical pattern recognition. Journal Royal Statistical Society B, 64:1-12, 2002.

C.C. Holmes and N.M. Adams. Likelihood inference in nearest-neighbour classification models. Biometrika, 90(1):99-112, 2003.

J.D. Knowles and D. Corne. Approximating the Nondominated Front Using the Pareto Archived Evolution Strategy. Evolutionary Computation, 8(2): 149-172, 2000.

M.A. Kupinski and M.A. Anastasio. Multiobjective Genetic Optimization of Diagnostic Classifiers with Implications for Generating Receiver Operating Characterisitic Curves. IEEE Transactions on Medical Imaging, 18(8):675685, 1999.

D. Mossman. Three-way ROCs. Medical Decision Making, 19(1):78-89, 1999.

F. Provost and T. Fawcett. Analysis and visualisation of classifier performance: Comparison under imprecise class and cost distributions. In Proceedings of the Third International Conference on Knowledge Discovery and Data Mining, pages 43-48, Menlo Park, CA, 1997. AAAI Press.

F. Provost and T. Fawcett. Robust classification systems for imprecise environments. In Proceedings of the Fifteenth National Conference on Artificial Intelligence, pages 706-7, Madison, WI, 1998. AAAI Press.

B.D. Ripley. Neural networks and related methods for classification (with discussion). Journal of the Royal Statistical Society Series B, 56(3):409456, 1994.

M.J.J. Scott, M. Niranjan, and R.W. Prager. Parcel: feature subset selection in variable cost domains. Technical Report CUED/F-INFENG/TR.323, Cambridge University Engineering Department, 1998.

A. Srinivasan. Note on the location of optimal classifiers in n-dimensional ROC space. Technical Report PRG-TR-2-99, Oxford University Computing Laboratory, Oxford, 1999.

D. Van Veldhuizen and G. Lamont. Multiobjective Evolutionary Algorithms: Analyzing the State-of-the-Art. Evolutionary Computation, 8(2):125-147, 2000.

X. Yao, Y. Liu, and G. Lin. Evolutionary Programming Made Faster. IEEE Transactions on Evolutionary Computation, 3(2):82-102, 1999.

M.H. Zweig and G. Campbell. Receiver-operating characteristic (ROC) plots: a fundamental evaluation tool in clinical medicine. Clinical Chemistry, 39: 561-577, 1993. 